\title{
Light scattering influence in cyanobacteria suspensions inside a photobioreactor
}

\author{
F. Fanjul-Vélez*, J. L. Arce-Diego* \\ Applied Optical Techniques Group, TEISA Department, University of Cantabria \\ Av de los Castros s/n, 39005 Santander (Spain)
}

\begin{abstract}
The application of biotechnology is increasing in areas such as agriculture, biochemistry or biomedicine. Growing bacteria or algae could be beneficial for supplying fuel, drugs, food or oxygen, among other products. An adequate knowledge of biological processes is becoming essential to estimate and control products production. Cyanobacteria are particularly appropriate for producing oxygen and biomass, by consuming mainly carbon dioxide and light irradiation. These capacities could be employed to provide human subsistence in adverse environments, as basic breathing and food needs would be satisfied. Cyanobacteria growing is carried out in bioreactors. As light irradiation is quite relevant for their behavior, photobioreactors are needed. Photobioreactors are designed to supply and control the amounts of elements they need, in order to maximize growth. The adequate design of photobioreactors greatly influences production throughput. This design includes, on the optical side, optical illumination and optical measurement of cyanobacteria growth. The influence of optical scattering is fundamental for maximizing cyanobacteria growing, as long as for adequately measure this growth. In this work, optical scattering in cyanobacteria suspensions is analyzed. Optical properties of cyanobacteria and its relationship with concentration is taken into account. Several types of cyanobacteria are considered. The influence of different beam spatial profiles and irradiances is studied by a Monte Carlo approach. The results would allow the consideration of the influence of optical scattering in the detected optical signal employed for growth monitoring, as a function of cyanobacteria type and optical beam parameters.
\end{abstract}

Keywords: cyanobacteria, photobioreactor, light scattering, optical illumination, photochemical effect

\section{INTRODUCTION}

The applications of biotechnology are nowadays increasing in areas such as agriculture or biochemistry, and even biomedicine. The benefits of microorganisms comprise fuel, drugs, food or even oxygen supply, among other products. Knowledge on biological processes is becoming essential in order to be able to adequately estimate and control the production of these elements. Several microorganisms are employed, and their intrinsic characteristics are highly varying. Cyanobacteria are a particular type of microorganisms with the capability of producing oxygen and biomass, where they are exposed to $\mathrm{CO}_{2}$ and light irradiation. Therefore, they could be fundamental for human subsistence in adverse environments, as basic needs of breathing and food would be guaranteed. Their behavior is of utmost relevance for maximizing relevant elements production [1].

Cyanobacteria growth is controlled and potentiated, as other microorganisms, in the so-called bioreactors [2]. These devices are specifically designed to favor microorganisms growth, by supplying and controlling the elements they need. In the particular case of cyanobacteria, light irradiation is fundamental for their growth, and the devices are then called photobioreactors. The adequate design of photobioreactors greatly influences elements production throughput. This design includes optical illumination and optical measurement of cyanobacteria growth.

In this work, optical scattering in cyanobacteria suspensions is analyzed. Optical properties of cyanobacteria and its relationship with concentration is taken into account. Several types of cyanobacteria are considered. The influence of different beam spatial profiles and irradiances is studied by a Monte Carlo approach. The results would allow the consideration of the influence of optical scattering in the detected optical signal employed for growth monitoring, as a function of cyanobacteria type and optical beam parameters.

*fanjulf@unican.es; arcedj@,unican.es; phone +34 942206730; www.teisa.unican.es/toa

Optical Interactions with Tissue and Cells XXIX, edited by E. Duco Jansen, Hope Thomas Beier, Proc. of SPIE Vol. 10492, 104920K · C 2018 SPIE · CCC code: 1605-7422/18/\$18 · doi: 10.1117/12.2290173 


\section{ESSENTIALS OF PHOTOBIOREACTORS}

The design of photobioreactors involves several key elements. Among the most relevant ones is optical irradiation. Some photobioreactors employ solar light, but it is difficult to control. Typically, solar light can suffer from unequal exposition to all the bacteria, low area to volume ratios, slow mass transference and inefficiency. Cyanobacteria are usually grown with LED optical sources or fluorescent lamps. Light irradiation depends on source to bioreactor distance, cyanobacteria concentration or the biofilm formation. Uneven illumination makes some cyanobacteria stop the photosynthesis process. Optical illumination spectrum is also quite relevant. Usually optimum wavelengths are in the range from 400 to $700 \mathrm{~nm}$. Irradiance also greatly influences the efficiency of the process. An insufficient irradiance results in growth decreasing, while an irradiation excess may provoke photo inhibition. The shielding effect of surface cyanobacteria with respect to deep cyanobacteria has to be considered [3]. Photobioreactors usually comprise a stirring system to cope with this problem. Although a stirrer makes every cyanobacteria be exposed to light irradiation, it also imposes a non-continuous irradiation. This aspect should be also taken into account. $\mathrm{CO}_{2}$ supply is also needed for cyanobacteria growth. It is usually introduced in the photobioreactor by means of continuous injection at the bottom of the sample. The amount of $\mathrm{CO}_{2}$ supply is also a relevant parameter for the efficiency of the process. Even $\mathrm{pH}$ should be controlled in order to maintain the appropriate conditions for cyanobacteria growth.

The basis of the production process in cyanobacteria is oxygenic photosynthesis [1]. Water in the bioreactor is used as an electrons donor in combination with $\mathrm{CO}_{2}$, and oxygen $\mathrm{O}_{2}$ is freed. The photosynthesis process can be divided into two phases, the light-dependent and light-independent ones. The first phase converts light in chemical energy. Light is captured by photoactive pigments, mainly chlorophylls and carotenoids [2]. The energy generated is supplied to proteins by means of photosystem I (centered at $700 \mathrm{~nm}$ ) and photosystem II (centered at $680 \mathrm{~nm}$ ). This phase generates $\mathrm{O}_{2}$. In the dark phase the new produced substances contribute to the generation of organic compounds, such as glucose, in the Calvin cycle. Photosynthetic efficiency is governed by irradiance and wavelength $[4,5,6]$, temperature (with an approximate double increase every $10^{\circ} \mathrm{C}$, until damage), $\mathrm{CO}_{2}$ concentration and $\mathrm{O}_{2}$ concentration. Cyanobacteria usually contain just chlorophyll a. This and other compounds give them a characteristic blue or green color. There are also red cyanobacteria. Typically light in visible wavelength is transferred to chlorophyll a reaction center. The considerations exposed in this section give rise to the relevance of light irradiation on cyanobacteria. The irradiance, wavelength and spatial light distribution are of utmost importance for the efficiency of the process.

A photobioreactor was built in the frame of this work. This photobioreactor presents four independent cells for cyanobacteria growth. Both illumination light and probing light can be controlled. Therefore, the influence of irradiance or spectrum can be analyzed, and it is also possible to implement different approaches for cyanobacteria growth monitoring. Figure 1 shows a detail of the photobioreactor.

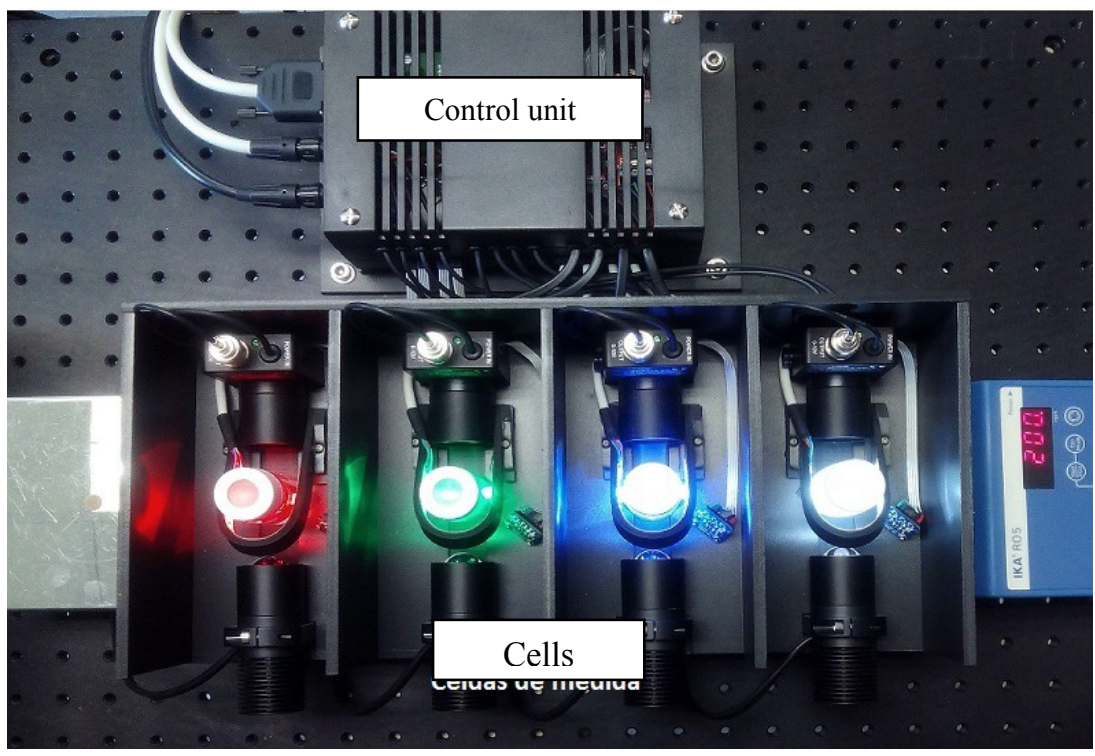

Figure 1. Photobioreactor employed in this work, with the four cells for cyanobacteria growth. 


\section{RESULTS AND DISCUSSION}

The analysis of the present problem requires the calculation of the distribution of light in a three-dimensional sample. This objective is reached by means of the Radiation Transport Theory (RTT). The model assumes that the scattering events are sufficiently numerous as to the light to be considered incoherent, in such a way that polarization or interference effects can be neglected. As a consequence, the basic parameter of light is the specific intensity, $I(\vec{r}, \hat{s}, t)$ $\left(W / m^{2} s r\right)$, that is, the light power per unit area per unit solid angle. The radiation is expected to be at point $\vec{r}$, and to follow the $\hat{s}$ direction. The scattering events are treated according to the scattering phase function, $p\left(\hat{s}, \hat{s}^{\prime}\right)$, which contains the probabilities of light to be scattered in the different directions. Light comes from direction $\hat{S}^{\prime}$ and is redirected to $\hat{s}$. The basic idea in order to write the temporal differential radiation transport equation is that radiation from a particle attenuates due to absorption and scattering and also gains power because another particle can scatter light in the direction of the particle of interest. This can be written as

$$
\frac{1}{c_{m}} \frac{d}{d t} I(r(t), \hat{s}, t)=-\left(\mu_{a}+\mu_{s}\right) I(r(t), \hat{s}, t)+\frac{\mu_{s}}{4 \pi} \int p\left(\hat{s}, \hat{s}^{\prime}\right) I\left(r\left(t^{\prime}\right), \hat{s}^{\prime}, t\right) d \Omega^{\prime}+Q(r(t), \hat{s}, t) .
$$

In equation (1), $c_{m}$ is the speed of light in the medium, $\Omega$ refers to the solid angle and $Q(r(t), \hat{s}, t)$ represents a source placed at the point of interest. For our purposes, there are no sources inside the tissue and a situation of steadystate can be considered. In this case, $\frac{d}{d t} I(r(t), \hat{s}, t)=0$ and $Q(r(t), \hat{s}, t)=0$. With this assumption and rewriting equation (1) a bit, we obtain

$$
\hat{\mathrm{s}} \cdot \bar{\nabla} \mathrm{I}(\mathrm{r}, \hat{\mathrm{s}})=-\left(\mu_{\mathrm{a}}+\mu_{\mathrm{s}}\right) \mathrm{I}(\mathrm{r}, \hat{\mathrm{s}})+\frac{\mu_{\mathrm{s}}}{4 \pi} \int_{4 \pi} \mathrm{p}\left(\hat{\mathrm{s}} \cdot \hat{\mathrm{s}}^{\prime}\right) \mathrm{I}\left(\mathrm{r}, \hat{\mathrm{s}}^{\prime}\right) \mathrm{d} \Omega^{\prime}
$$

An analytical solution can be given for this equation. The main attempts try to simplify the problem by making approaches in limiting cases, such as absorption-dominant limit or diffusion approximation, depending on the relative importance of absorption and scattering events in a particular biological tissue. However, there is another possibility to solve the equation, using a numerical approximation. Numerical analysis has been widely applied to a great variety of problems governed by differential equations. In the concrete topic of the radiation transport equation, the Monte Carlo method has demonstrated its applicability and accuracy, compared with exact solutions $[7,8]$. The key point is the inclusion of the random character on a computer, by means of a mathematical probability analysis, in such a way that numbers with any probability distribution can be obtained from numbers that follow a uniform distribution between 0 and 1. Light is treated as a sequence of photons, whose number is intended to be representative of the accuracy of the solution obtained. One photon is launched and its trajectory, affected by scattering, and loose of energy are calculated, while the absorption in each point is stored. Cylindrical symmetry is assumed, because laser beams usually show this kind of symmetry, so in fact the data can be interpreted as coming from a 3-D analysis. The complete tissue is divided in a two-dimensional grid in the $\mathrm{r}$ and $\mathrm{z}$ directions. As usual, more accurate results require a smaller grid, but the need of a reduced time of computation imposes a limit.

The program calculates several parameters. From the point of view of this study, the most important one is the absorption of each element of the grid. The fluence rate is also showed, and the reflectance and transmittance as a function of the radial position and the angle of observation. One significant consideration is worthwhile. The Monte Carlo program assumes that the optical beam is infinitely narrow, and that it has perpendicular incidence. The second assumption is reasonable, but the former can provoke serious disappointments with the reality, specially if the dimensions of the optical spot and the tissue are of the same order. In order to correct this limitation, another program by the same authors ${ }^{10}$ implements the convolution of the results. In this way, the solution of the Monte Carlo analysis can be later transformed for taking into account cylindrical or gaussian geometry of the laser beam. 
This implementation of the Monte Carlo model is also multi-layered, so it is possible to define several layers of different materials, with their borders always perpendicular to the laser beam, which is very useful due to tissues usually can be divided in different strata. For the appropriate definition of the model, the characteristics and dimensions of each layer are required. The dimensions of the structure of vocal cords were obtained before. The optical parameters needed are the index of refraction, $n$, the absorption coefficient, $\mu_{a}$, the scattering coefficient, $\mu_{s}$, and the anisotropy of scattering, $g$. This last parameter is called average cosine of scatter (dimensionless), and is related with the scattering phase function by the relationship

$$
g=\frac{\int_{4 \pi} p\left(\hat{s} \cdot \hat{s}^{\prime}\right) \hat{s} \cdot \hat{s}^{\prime} d \Omega^{\prime}}{\int_{4 \pi} p\left(\hat{s} \cdot \hat{s}^{\prime}\right) d \Omega^{\prime}}=\frac{1}{4 \pi W_{o}} \int_{4 \pi} p\left(\hat{s} \cdot \hat{s}^{\prime}\right) \hat{s} \cdot \hat{s}^{\prime} d \Omega^{\prime}=\frac{1}{2 W_{o}} \int_{4 \pi} p(\cos \theta) \cos \theta \sin \theta d \theta,
$$

where the albedo is defined by

$$
W_{o}=\frac{1}{4 \pi} \int_{4 \pi} p\left(\hat{s} \cdot \hat{s}^{\prime}\right) d \Omega^{\prime}=\frac{\sigma_{s}}{\sigma_{a}+\sigma_{s}}=\frac{\mu_{s}}{\mu_{a}+\mu_{s}} .
$$

The average cosine of scatter gives an idea about the probability of being scattered in a particular direction. For instance, $g=0$ implies that all directions all equally probable. If $g>0$ the radiation tends to be scattered forward, and vice versa. The albedo tries to illustrate the predominance of absorption or scattering in a particular tissue.

The results were obtained on A. cylindrica. The application of the previously given theory in terms of incoherence is assumed [9]. The properties of this particular kind of cyanobacteria, particularly absorption and scattering coefficients, anisotropy of scattering and refractive index, were measured for different concentrations [10]. Other anisotropic effects are neglected [11]. In the approach, several wavelengths were considered: 405, 490, 530, 625 and $730 \mathrm{~nm}$, and also different concentrations. The optical beam traversing the sample was assumed to be either Gaussian or top-hat [12], with a radius between 0.1 and $0.5 \mathrm{~cm}$. These conditions correspond to a realistic scenario in our photo-biorreactor.

Figure 2 shows an example of optical spatial irradiation profiles that traverses the sample.

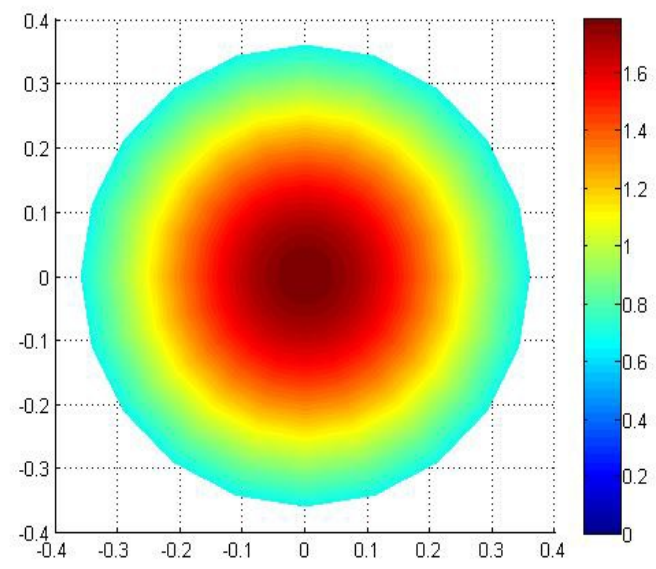

a)

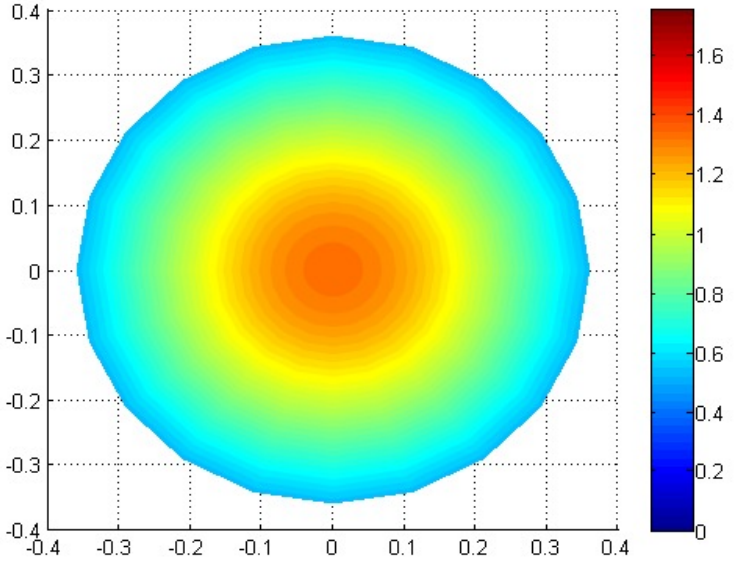

b)

Figure 2. Transmitted optical radiation distribution through the photobioreactor with cyanobacteria at a) $0.202 \mathrm{~kg} / \mathrm{m}^{3}$ and b) $0.431 \mathrm{~kg} / \mathrm{m}^{3}$ concentration, for a Gaussian $0.5 \mathrm{~cm}$ radius beam at $730 \mathrm{~nm}$. 
Figure 2 shows the great influence of concentration in optical transmission, which diminishes with concentration, as expected. This fact can be employed in order to estimate cyanobacteria growth, as a greater concentration will imply a decrease in optical transmission.

The evolution of the total optical fluence as a function of concentration and optical wavelength, for the case of a a radius of $0.1 \mathrm{~cm}$, can be seen in Figure 3 .

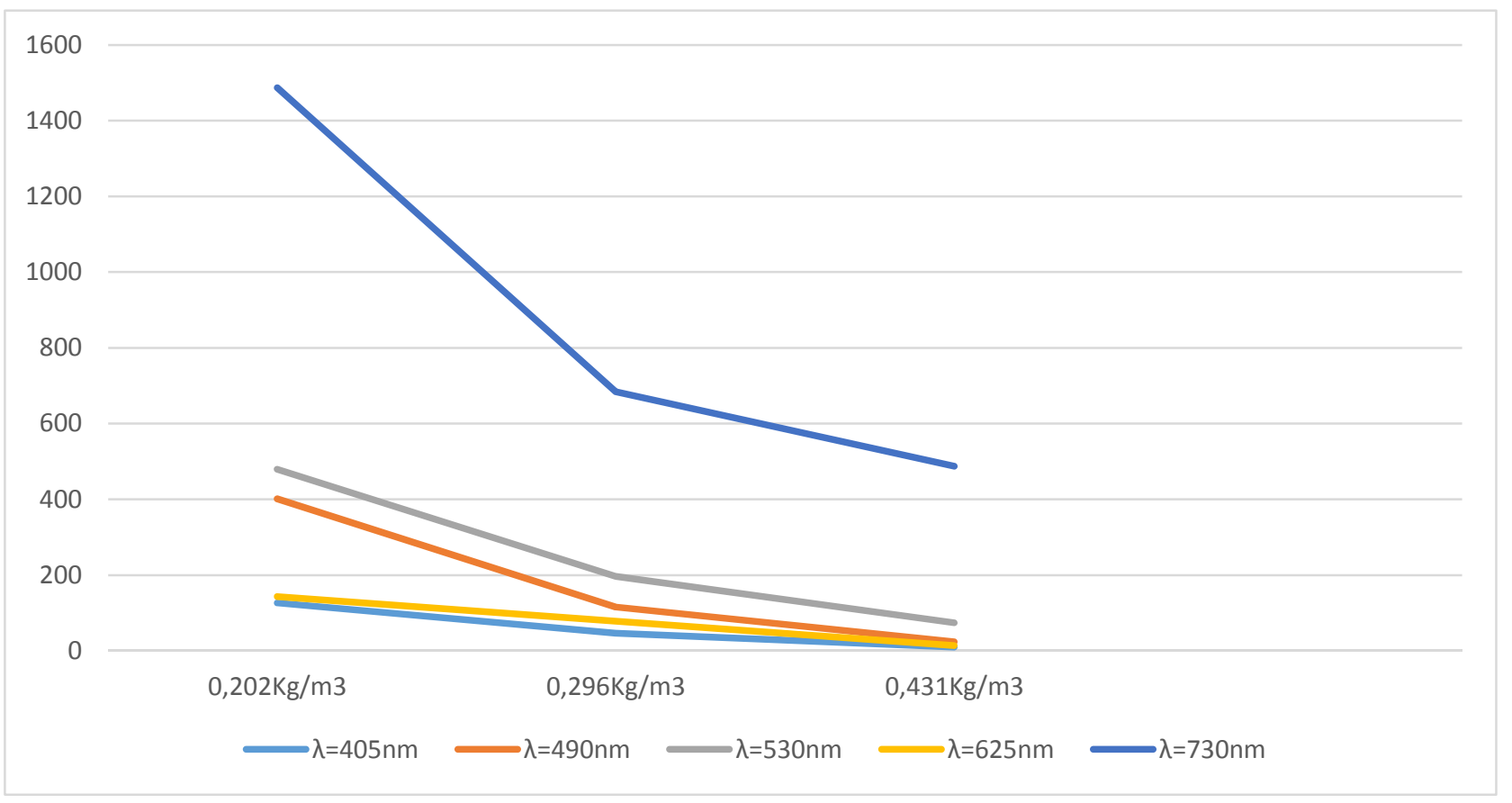

Figure 3. Total optical fluence after traversing the photobioreactor at different concentrations and wavelengths, for a Gaussian $0.1 \mathrm{~cm}$ radius beam.

Figure 3 stresses the difference in absorption and scattering coefficients of cyanobacteria, as a function of wavelength. In general, a decrease in wavelength is associated with an absorption increase, although there are some values $(625 \mathrm{~nm})$ with an abnormal increased absorption. This fact is in line with the considerations on spectrum discussed in section 2.

The transmission decrease is not linear with the concentration, as scattering plays a relevant role in the problem. This fact implies that an accurate growth estimator would need to pay attention to this issue.

\section{CONCLUSIONS}

Biotechnology is recently focused on production of elements. The efficiency of this production relies on the design of the so-called bioreactors. Cyanobacteria are a particular case in biotechnology, as they are capable of producing oxygen and biomass. These two products would be essential for guaranteeing human sustainability in adverse environments. The main characteristics of cyanobacteria growth have been exposed. As there is a great influence of optical irradiation, this work has focused on light illumination distribution, as a function of wavelength, irradiance or spatial profile. All of them greatly influence the photochemical process.

A photobioreactor was built in the frame of the present work. Several concentrations of cyanobacteria have been considered, irradiated by optical radiation with different spatial profiles, wavelengths and radii. The results show great differences as a function of cyanobacteria concentration, and the non-linearity caused by scattering of cyanobacteria. The results of the Radiation Transport Theory and Monte Carlo approaches can be employed in the design of photobiorreactors. The objective would be double, as estimated growth measurement accuracy could be employed, as long as the efficiency of the process could be optimized. 


\section{ACKNOWLEDGEMENTS}

This work has been partially supported by the project "New active phases in transition metals and rare earth nano-oxides stabilized at high pressure" (MAT2015-69508-P) of the Spanish Ministry of Economy and Competitiveness, cofunded by FEDER funds, and by the San Cándido Foundation.

\section{REFERENCES}

[1] Demmig-Adams, B., "Carotenoids and photoprotection in plants. A role for the xanthophyll zeaxanthin," Biochim Biophys Acta 1020, 1-24 (1990).

[2] Wojtasiewicz, B. and Stoń-Egiert, J., "Bio-optical characterization of selected cyanobacteria strains present in marine and freshwater ecosystems," J Appl Phycol 28(4), 2299-2314 (2015).

[3] Salas-García, I., Fanjul-Vélez, F. and Arce-Diego, J. L., "Photosensitizer absorption coefficient modelling and necrosis prediction during photodynamic therapy," Journal of Photochemistry and Photobiology B: Biology 114, 79-86 (2012).

[4] Salas-García, I., Fanjul-Vélez, F. and Arce-Diego, J. L., "Superficial radially-resolved fluorescence and threedimensional photochemical time-dependent model for Photodynamic Therapy," Optics Letters 39, 1845-1848 (2014).

[5] Salas-García, I., Fanjul-Vélez, F. and Arce-Diego, J. L., "Spatial photosensitizer fluorescence emission predictive analysis for photodynamic therapy monitoring applied to a skin disease," Optics Communications 285, 1581-1588 (2012).

[6] Salas-García, I., Fanjul-Vélez, F. and Arce-Diego, J. L., "Influence of the human skin tumor type in Photodynamic Therapy analysed by a predictive model," International Journal of Photoenergy 2012, 75920517592059 (2012).

[7] Wang, L., Jacques, S. L. and Zheng, L., "MCML - Monte Carlo modeling of light transport in multi-layered tissues," Computer Methods and Programs in Biomedicine 47, 131-146 (1995).

[8] Fanjul-Vélez, F. and Arce-Diego, "Modeling thermotherapy in vocal cords novel laser endoscopic treatment," Lasers in Medical Science 23(2), 169-177 (2008).

[9] Ortega-Quijano, N., Fanjul-Vélez, F., de Cos-Pérez, J. and Arce-Diego, J. L., "Analysis of the depolarizing properties of normal and adenomatous polyps in colon mucosa for the early diagnosis of precancerous lesions," Optics Communications 284, 4852-4856 (2011).

[10]Heng, R. L., Lee, E. and Pilon, L., "Radiation characteristics and optical properties of filamentous cyanobacterium Anabaena cylindrica," J. Opt. Soc. Am. A31, 836-845 (2014).

[11] Ortega-Quijano, N., Fanjul-Vélez, F. and Arce-Diego, J. L., "Polarimetric study of birefringent turbid media with three-dimensional optic axis orientation," Biomedical Optics Express 5, 287-292 (2014).

[12]Fanjul-Vélez, F., Salas-García, I. and Arce-Diego, J. L., "Analysis of laser surgery in non-melanoma skin cancer for optimal tissue removal," Laser Physics 25, 025606-1, 8 (2015). 